\title{
URBAN MOBILITY AND QUALITATIVE RESEARCH IN HISTORIC PLACES
}

\author{
ALEJANDRO ACOSTA COLLAZO \\ Habitat Design Department, Autonomous University of Aguascalientes, Mexico
}

\begin{abstract}
Mobility is a critical factor of urban development and the cause of frequent changes in city infrastructure and in public spaces. The study of mobility in historic places and landmarks in the city of Aguascalientes, using qualitative research methods, helped to understand the incremental loss of heritage architecture in the last decades. There are several reasons to visit a historic site and tourism levels have increased lately in Mexico. Besides the importance of tourism, there are people who go to work there, some others attend schools, and a few of them live in the historic centre. The analysis of parking lots inventory wasn't enough to understand mobility in historic places. It was through the design and implementation of a survey, using a qualitative statistical method, that research determined the origin of visitors, visiting times, level of education, transportation means and the way people use historic places. Furthermore, such research method determined the relationship of these factors by a multiple correspondence analysis. This analysis helped in reconstructing relevant information about such visitors. The qualitative was obtained from a printed questionnaire in person. The data clarified patterns of relationships and correspondence among variables. So the perception of the visitors showed the effects of the mobility in historic places. Tourism and mobility should foster preservation ethics nowadays. Additionally, it's possible to promote the use of less polluting transportation in historic places. It's achievable to design and install a light rail transit system (LRT) into the historic centre. For example, a good design could include an optimum track alignment for a LRT corridor on Madero Av. in the city of Aguascalientes, from the principal square to the main railroad station. This also could improve a sustainable development in the near future.
\end{abstract}

Keywords: urban transport, historic preservation, mobility, public spaces.

\section{INTRODUCTION}

Historic places are essential for city administrators and most of the time they are included in city planning for preservation. However, there are factors like mobility that impact somehow streetscapes and infrastructures. Specialized preservation of public spaces can improve quality of life. So, architects, city planners, designers, etc. should be aware of new paradigms in design and restoration techniques. Advanced visions of sustainable transport and the analysis of social opinions can be a good help in the search for good urban design practices.

Several international journals mention topics about transport development, application and integration. The use of new technologies, logistics and optimisation, are also subjects of interest. Likewise, social factors are in the scope of several papers. The research strategy used in the writing of this paper is facing the mobility challenges from a social perspective. An ethnographic method was helpful to find out -through observation- how visitors of public spaces used them. But also, it was convenient to obtain information from a printed questionnaire in person.

\section{METHODOLOGY}

Active observation is significant in the methodology of studying historic places, but in this case the design of a survey complemented the inductive emphasis of it. Face to face questionnaires were useful to understand the way people arrived to the historic centre; particularly to find out where they parked or their means of transportation. Since the main goal of the paper was to observe how mobility has been changing historic places, specifically 
the historic centre of Aguascalientes in the last few years, the parking lots became essential. But it was necessary to design and apply a survey to understand the problem of loss of heritage buildings due to mobility. A questionnaire, with the following goals, was designed to: 1 . Detect the gender of the interviewed person who visits public spaces in the city centre; 2 . Find out the origin of the visitors (local, national or foreigner); 3 . Discover how much time visitors spend in public spaces; 4 . Identify the level of education of the visitors; 5 . Find out the means of transportation of the visitors; 6 . Detect the places the visitors usually park at, to get to public spaces.

In order to reach the final step in the methodology (validation of results and discussion) it was useful to set the methodological proceedings in the research. In this case, it was helpful to start formulating a problem. So, to understand the loss of historic buildings in the core of the city it was convenient to understand if mobility patterns had to do with it. Incidentally, it's always useful to apply qualitative methods if the opinions of people are going to be analysed. A conceptual map was designed to resolve the problem just mentioned (see Fig. 1).

Statistical methods also include qualitative alternatives focusing on urban issues as preservation of historic buildings. The selected variables for this research were: gender; origin of visitors; time spent in public spaces; education level; means of transportation and the place people chose to park. The sample size was based on a questionnaire (see Fig. 2), applied in four emblematic places in the historic centre, with a sample of 20 people in each place. The collected information about the population of interest was processed in tables using a Burt's matrix. Camiz and Gomes argue that Multiple Correspondence Analysis (MCA) is the best-known exploratory factor analysis method to deal with it [1]. The categories of variables were particularly useful for registering urban mobility actions of the public space users, including some other hidden factors. An original idea was to use a GPS program to understand urban mobility, but this would've changed the qualitative side of the research. After obtaining the results the discussion was required to support the assumptions of this paper. Also, innovations to currently urban mobility can be pursued with new researches, and furthermore, to support cultural heritage sustainability.

\section{RESULTS}

One of the most visited historic buildings of the city is the main Cathedral. It's located at the main square in the historic core. $60 \%$ of the inquired visitors were female from Aguascalientes. Half of the women visitors were from 18 to 60 years old. Some of the visitors (tourists) not only go to the place to pray, but to visit the building. No foreigners were detected during this research, but the visitors who were more interested in the Cathedral came from other states than Aguascalientes. This is, more women than men. Also 40\% of the visitors were old people who spent more time in the historic place (see Table 1).

The visiting time at the place was basically medium, but adult women preferred to stay longer than men. Visitors were basically local. The education level of visitors was more frequent in the high school category. No visitors with postgraduate levels were detected.

The National Museum of Death has been a whole attraction in the city of Aguascalientes since it was inaugurated in 2007. It includes a collection of artistic objects, related to funerary art, iconography and symbols of death. There's been a considerable interest in the Mexican particular vision of the exposed objects. The collected data obtained from the questionnaires were distributed in a matrix (see Table 2). Since the methodology to obtain the Burt's table is shown in Table 1, for practical reasons in the writing of this paper, only the results of the Burt's table are included in Tables 2, 3 and 4. 


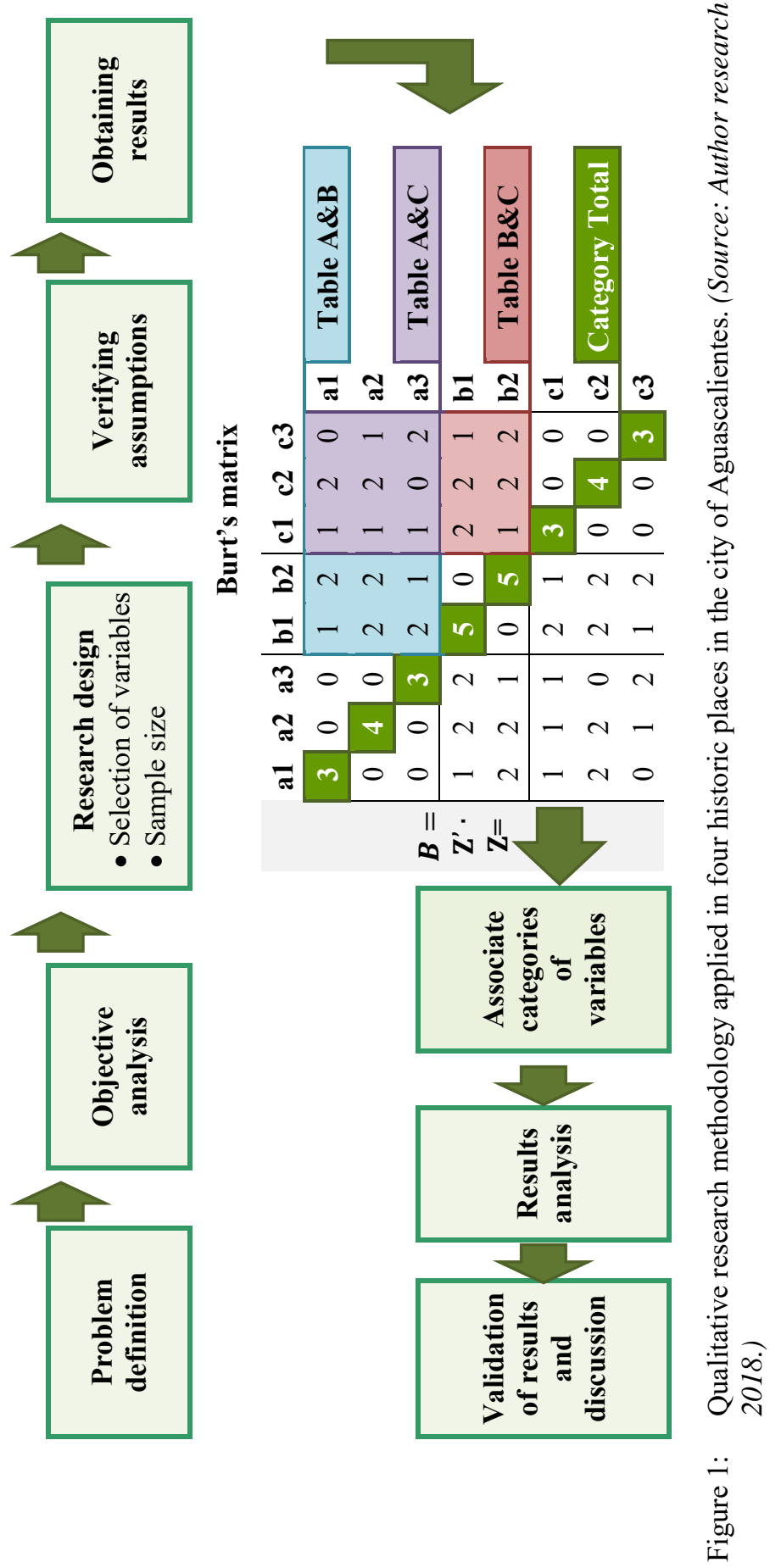




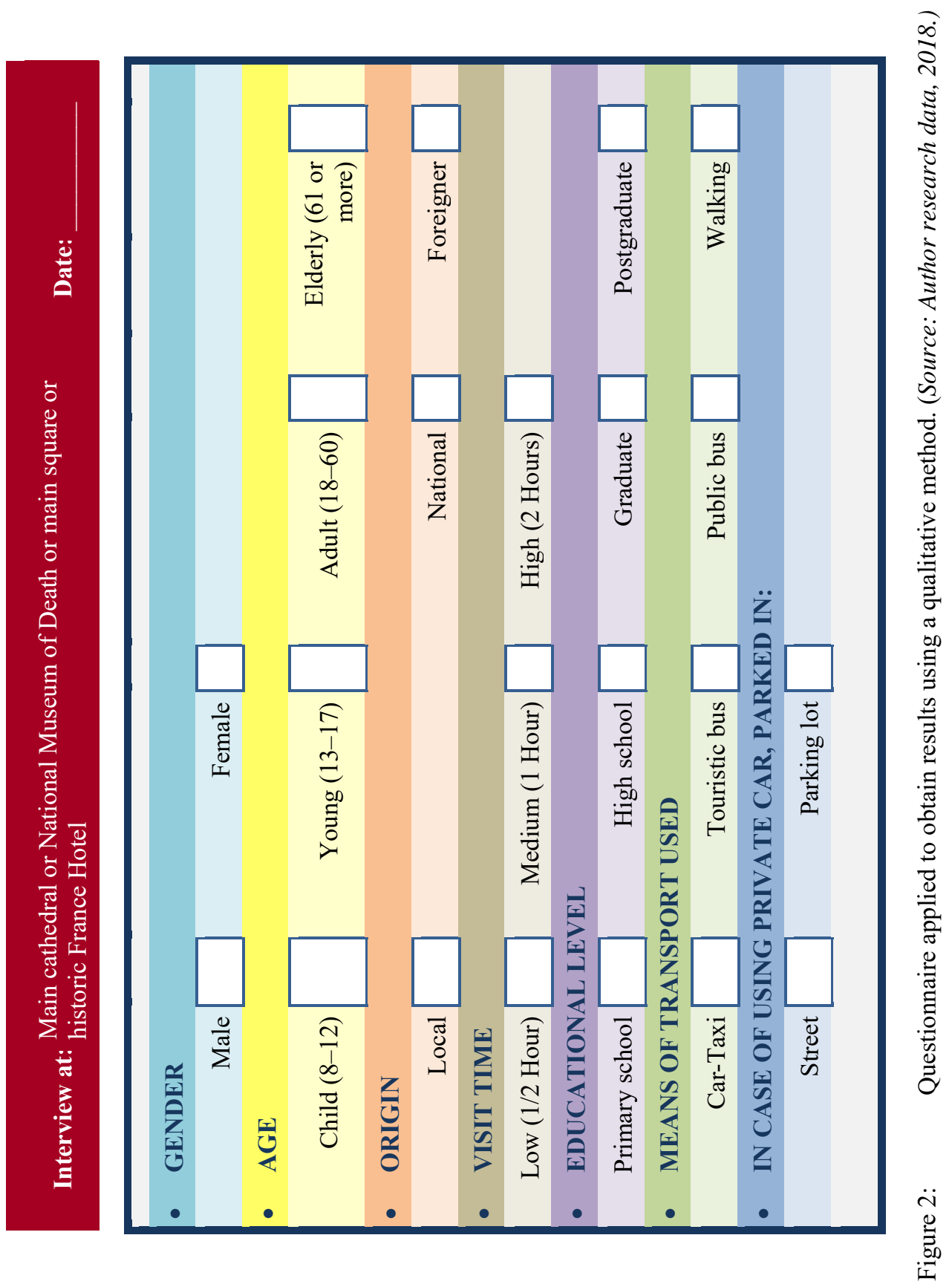




\begin{tabular}{|c|c|c|c|c|c|c|c|c|c|c|c|c|c|c|c|c|c|c|c|c|c|c|c|}
\hline & \begin{tabular}{l|}
$\ddot{g}$ \\
$\ddot{g}$
\end{tabular} & $\begin{array}{c}0 I \\
\text { ou!y.ग6d }\end{array}$ & בี & 0 & 0 & 0 & 0 & 0 & 0 & - & 0 & 0 & 0 & 0 & 0 & 0 & - & 0 & 0 & 0 & -1 & 0 & $0 \mathrm{~m}$ \\
\hline & 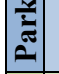 & 1әว.11S & ב & 0 & 0 & 0 & 0 & 0 & 0 & 0 & 0 & -1 & 0 & 0 & 0 & 0 & 0 & -10 & 0 & 0 & 0 & 0 & $0 \mathrm{~N}$ \\
\hline & 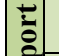 & อ̊u!Yा火 & 吉 & 0 & 0 & 0 & - & - & 0 & 0 & 0 & 0 & 0 & 0 & 0 & 0 & 0 & 0 & 0 & - & 0 & 0 & $0 \mathrm{~m}$ \\
\hline & 坣 & snq & bn & 一 & - & - & 0 & 0 & 0 & 0 & - & 0 & o & -1 & -1 & -1 & 0 & 0 & 0 & 0 & o & -1 & $0 \infty$ \\
\hline & 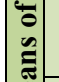 & $\begin{array}{c}\text { snq } \\
\text { ग!̣!!.Ino } L\end{array}$ & bo & 0 & 0 & 0 & 0 & 0 & 0 & 0 & 0 & 0 & 0 & 0 & 0 & 0 & 0 & 0 & 0 & 0 & 0 & 0 & 100 \\
\hline & $\sum$ & !X姃 & $\overrightarrow{60}$ & 0 & 0 & 0 & 0 & 0 & - & - & 0 & - & - & 0 & 0 & 0 & - & -1. & - & 0 & - & 0 & $-a$ \\
\hline & $\begin{array}{l}\overline{0} \\
\text { 르 }\end{array}$ & $\begin{array}{c}\text { әрепре.і8 } \\
-150 d\end{array}$ & 进 & 0 & 0 & 0 & 0 & 0 & 0 & 0 & 0 & 0 & 0 & 0 & 0 & 0 & 0 & 0 & 0 & 0 & 0 & 0 & 100 \\
\hline & ב⿱艹 & әұвпрв.ID & $\notin$ & 0 & 0 & 0 & - & 一 & 0 & -1 & 0 & 0 & 0 & 0 & 0 & 0 & 0 & 0 & 0 & 0 & - & 0 & $0 \quad 4$ \\
\hline & | & $\begin{array}{c}\text { [00पगS } \\
\text { पภ̊!!H }\end{array}$ & $\notin$ & 0 & 0 & - & 0 & 0 & -1 & 0 & - & 0 & -1 & 0 & -1 & 0 & - & -1. & -1 & 0 & 0 & -1 & -1 으 \\
\hline 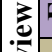 & 지 & K.IV & 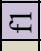 & 一 & - & 0 & 0 & 0 & 0 & 0 & 0 & - & 0 & - & 0 & - & 0 & 010 & 0 & - & 0 & 0 & 00 \\
\hline 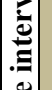 & 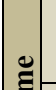 & $\begin{array}{c}(\operatorname{s.InoH} z) \\
\text { पo̊!! }\end{array}$ & 3 & 0 & 0 & 0 & 0 & 0 & 0 & 0 & 0 & 0 & 0 & 0 & 0 & -1 & 0 & 0 & 0 & 0 & 0 & 0 & $0-$ \\
\hline : & 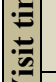 & $\begin{array}{l}\text { (.InoH I) } \\
\text { un!pəWW }\end{array}$ & ชิ & - & - & 0 & - & - & - & -1 & - & 0 & -1 & 0 & 0 & 0 & -1 & -1. & -1 & 0 & -1 & 0 & $-\cong$ \\
\hline $\mid$ & $>$ & $\begin{array}{c}\text { (InoH } \\
\text { Z/I)Mo' }\end{array}$ & $\overline{0}$ & 0 & 0 & - & 0 & 0 & 0 & 0 & 0 & -1 & 0 & -1 & -1 & 0 & 0 & 0 & 0 & - & 0 & -1 & 00 \\
\hline \begin{tabular}{|c|}
$\mathbf{U}$ \\
$\mathbf{N}$
\end{tabular} & & .ЈУ & శ & 0 & 0 & 0 & 0 & 0 & 0 & 0 & 0 & 0 & 0 & 0 & 0 & 0 & 0 & 0 & 0 & 0 & 0 & 0 & 00 \\
\hline . & 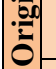 & [Euo & ชี & 0 & 0 & 0 & - & - & 0 & -1 & 0 & 0 & -1 & 0 & 0 & 0 & 一 & $-1-$ & -1 & 0 & -1 & 0 & $-a$ \\
\hline$\sum$ & & & 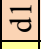 & 一 & - & - & 0 & 0 & - & 0 & - & - & 0 & - & - & - & 0 & 015 & 0 & - & 0 & - & $0=$ \\
\hline & & 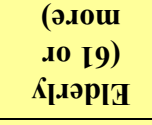 & ชิ| & - & 0 & 0 & 0 & 0 & - & 0 & 0 & 0 & 0 & -1 & 0 & -1 & - & 0. & - & - & 0 & 0 & -1 \\
\hline & $\mid \begin{array}{l}0 \\
0\end{array}$ & $\begin{array}{c}\text { (09-8I) } \\
\text { f[npV }\end{array}$ & $\overline{0}$ & 0 & - & 0 & - & - & 0 & -1 & - & 0 & -1 & 0 & 0 & 0 & 0 & -10 & 0 & 0 & -1 & 0 & 0 \\
\hline & & $\begin{array}{l}\text { (LI } \\
\mathbf{8} \mathbf{u} \\
\end{array}$ & ปี & 0 & 0 & -1 & 0 & 0 & 0 & 0 & 0 & 0 & 0 & 0 & -1 & 0 & 0 & 0 & 0 & 0 & 0 & -1 & $0 \mathrm{~m}$ \\
\hline & & $\begin{array}{l}(Z I-8) \\
\text { pI!पว }\end{array}$ & 잉 & 0 & 0 & 0 & 0 & 0 & 0 & 0 & 0 & -1 & 0 & 0 & 0 & 0 & 0 & 0 & 0 & 0 & 0 & 0 & $0-$ \\
\hline & : & әГвшәң & ฮี & - & - & 0 & - & 0 & - & - & - & 0 & -1 & 0 & -1 & 0 & - & -1. & - & 0 & 0 & 0 & $-\approx$ \\
\hline & 0 & $\mathbf{W}$ & त्) & 0 & 0 & - & 0 & - & 0 & 0 & 0 & -1 & 0 & -1 & 0 & -1 & 0 & 0 & 0 & - & - & - & $0 \infty$ \\
\hline & & 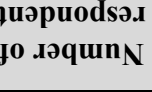 & & - & $N$ & $m$ & $\nabla$ & in & 0 & 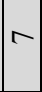 & $\infty$ & $a$ & 의 & 二 & 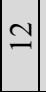 & $\approx$ & \pm & $\because$ & $\underset{-1}{0}$ & 二 & $\infty$ & 9 & 이 \\
\hline
\end{tabular}




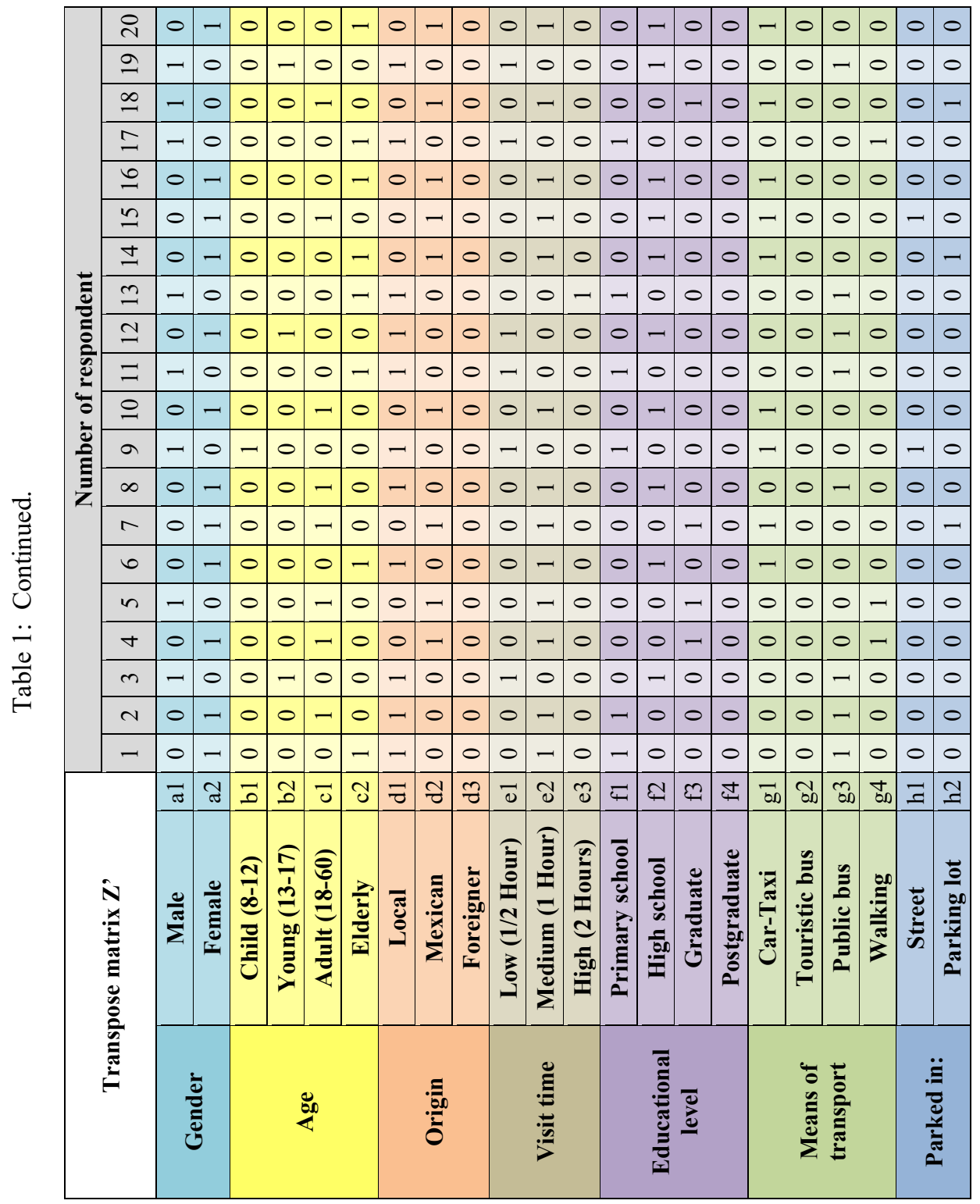




\begin{tabular}{|c|c|c|c|c|c|c|c|c|c|c|c|c|c|c|c|c|c|c|c|c|c|c|c|c|}
\hline \multirow{2}{*}{ 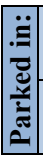 } & $\begin{array}{c}10 \mathrm{I} \\
\text { 8ิu!Y.16 d } \\
\end{array}$ & 고 & -1 & $N$ & 0 & 0 & $\sim$ & - & 0. & $m$ & 0 & 0 & $m$ & 0 & 0 & - & $N$ & 0 & $m$ & 0 & o & 0 & o.e & $m$ \\
\hline & Һәә..1S & $\exists$ & - & -1 & -1 & 0 & -1 & 0 & -1 & - & 0 & - & - & 0 & - & - & 0 & 0 & $N$ & 0 & 0 & 0 & N & 0 \\
\hline \multirow{4}{*}{ 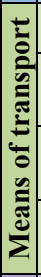 } & 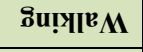 & 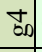 & $N$ & - & 0 & 0 & $\sim$ & - & $-1 c$ & $N$ & 0 & - & $N$ & 0 & - & 0 & $N$ & 0 & 0 & 0 & 0 & $m$ & 0 & 0 \\
\hline & $\begin{array}{c}\text { snq } \\
\text { ग!Iqnd }\end{array}$ & $\hat{\mathrm{bD}}$ & $\nabla$ & $\nabla$ & 0 & $m$ & $N$ & $m$ & $\infty$ & 0 & 0 & $\forall$ & $m$ & - & $\nabla$ & 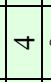 & 0 & 0 & 0 & 0 & $\infty$ & 0 & 0 & 0 \\
\hline & $\begin{array}{c}\text { snq } \\
\text { ग!ฺ!!.uno } L \\
\end{array}$ & ob & 0 & 0 & 0 & 0 & 0 & 0 & 0 & 0 & 0 & 0 & 0 & 0 & 0 & 0 & 0 & 0 & 0 & $\theta$ & 0 & 0 & 0 & 0 \\
\hline & !XE $\mathbf{L}^{-\boldsymbol{I}}$ & $\overline{00}$ & $N$ & $\sim$ & - & 0 & $\nabla$ & $\nabla$ & No & - & 0 & - & $\infty$ & 0 & - & 6 & $N$ & 0 & $a$ & 0 & 0 & 0 & N. & $m$ \\
\hline \multirow{4}{*}{ 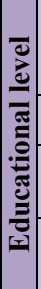 } & $\begin{array}{c}\text { әңєกрв.ภо } \\
\text {-150d }\end{array}$ & ष & 0 & 0 & 0 & 0 & 0 & 0 & 0 & 0 & 0 & 0 & 0 & 0 & 0 & 0 & 0 & $\theta$ & 0 & 0 & 0 & 0 & 0 & 0 \\
\hline & әұепре.ग & $\notin$ & $N$ & $N$ & 0 & 0 & $\nabla$ & 0 & 0 & $\nabla$ & 0 & 0 & $\nabla$ & 0 & 0 & 0 & $\nabla$ & 0 & $N$ & 0 & 0 & $N$ & 0 & $N$ \\
\hline & $\begin{array}{c}\text { I00पगS } \\
\text { पo̊!! }\end{array}$ & 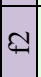 & $N$ & $\infty$ & 0 & $n$ & $m$ & $\nabla$ & $n$ & $n$ & 0 & $m$ & $r$ & 0 & 0 & 으 & 0 & 0 & 6 & 0 . & $\nabla$ & 0 & -1 & -1 \\
\hline & $\begin{array}{c}\text { I00чगs } \\
\text { Клвщ!!. }\end{array}$ & 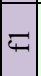 & $\nabla$ & $N$ & - & 0 & -1 & $\nabla$ & 6 & 0 & 0 & $m$ & $N$ & - & 0 & 0 & 0 & 0 & -1 & 0 . & t & - & - & 0 \\
\hline \multirow{3}{*}{ 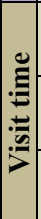 } & 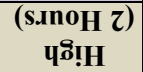 & 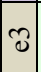 & - & 0 & 0 & 0 & 0 & - & -10 & 0 & 0 & 0 & 0 & - & - & 0 & 0 & 0 & 0 & 0 . & - & 0 & 0 & 0 \\
\hline & $\begin{array}{l}\text { (InoH I) } \\
\text { un!pәW }\end{array}$ & ชิ & $N$ & $=$ & 0 & 0 & $\infty$ & $\ln$ & $\nabla$ & a) & 0 & 0 & $\dddot{m}$ & 0 & $N$ & N & $\nabla$ & 0 & $\infty$ & 0 & $m$ & $N$ & -1 & $m$ \\
\hline & $\begin{array}{c}\text { (.InoH } \\
\text { z/I) MO T }\end{array}$ & $\overline{0}$ & $n$ & -1 & - & $m$ & 0 & $N$ & 0 & 0 & 0 & 6 & 0 & 0 & $m$ & $m$ & 0 & 0 & - & 0 . & $\nabla$ & - & - & 0 \\
\hline \multirow{3}{*}{ 告 } & 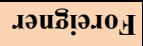 & $\eta$ & 0 & 0 & 0 & 0 & 0 & 0 & 0 & o & $\theta$ & 0 & 0 & 0 & 0 & 0 & 0 & 0 & 0 & 0 & 0 & 0 & 0 & 0 \\
\hline & [вuo!) $\mathbf{N}$ & ขี & $N$ & - & 0 & 0 & 0 & $m$ & 0 & a & 0 & 0 & $a$ & 0 & 0 & $n$ & $\nabla$ & 0 & $r$ & 0 & 0 & $N$ & - & $m$ \\
\hline & [ยวоT & 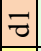 & 6 & in & - & $m$ & $N$ & in & $=$ & 0 & 0 & 6 & $\nabla$ & 一 & 6 & in & 0 & 0 & $N$ & 0 & $\infty$ & - & - & 0 \\
\hline \multirow{4}{*}{ 兽 } & $\begin{array}{c}\text { (ә.лош } \\
\text { Іо І9) } \\
\text { Кц.әрІЯ }\end{array}$ & ชิ & $m$ & in & 0 & 0 & 0 & $\infty$ & $n$ & $m$ & 0 & $N$ & in & - & $\nabla$ & $\nabla$ & 0 & 0 & $\nabla$ & 0 & $m$ & - & 0 & -1 \\
\hline & $\begin{array}{l}\text { (09-8I) } \\
\text { fInpV }\end{array}$ & $\overline{0}$ & $N$ & 0 & 0 & 0 & $\infty$ & 0 & $N$ & 6 & 0 & 0 & $\infty$ & 0 & - & $m$. & $\nabla$ & 0 & $\nabla$ & 0 & $N$ & $N$ & -1 & $\sim$ \\
\hline & $\begin{array}{l}(L I-\varepsilon I) \\
\text { ôunoג }\end{array}$ & ชิ & $N$ & - & 0 & $m$ & 0 & 0 & $m$ & 0 & 0 & $m$ & 0 & 0 & 0 & $m$ & 0 & 0 & 0 & 0 & $m$ & 0 & 0 & 0 \\
\hline & $\begin{array}{l}\text { (ZI-8) } \\
\text { рІ!Чว }\end{array}$ & D & - & 0 & - & 0 & 0 & 0 & -10 & 0 & 0 & - & 0 & 0 & - & 0 & 0 & 0 & - & 0 & 0 & 0 & - & 0 \\
\hline \multirow{2}{*}{ 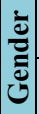 } & әбешән & สิ & 0 & $\approx$ & 0 & 一 & 6 & in & $n n$ & - & 0 & - & 二 & 0 & $N$ & $\infty$ & $\sim$ & 0 & $r$ & 0 & $\nabla$ & - & - & $\sim$ \\
\hline & 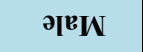 & $\pi$ & $\infty$ & 0 & - & $\sim$ & $N$ & $m$ & 0 & 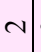 & 0 & $n$ & $N$ & - & $\nabla$ & $N$ & $\sim$ & 0 & N & 0 . & $\nabla$ & $N$ & - & - \\
\hline & \multirow{3}{*}{ 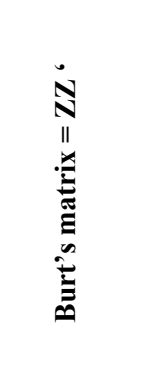 } & & $\pi$ & สี & D & ชิ & $\overline{0}$ & ชิ & $\approx$ & 김 & $\eta$ & $\overline{0}$ & ชี & 3 & $\varpi$ & 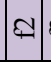 & 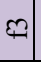 & 4 & $\overrightarrow{00}$ & के & 60 & & $\exists$ & ב \\
\hline & & & $\frac{0}{\Sigma}$ & 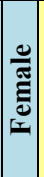 & 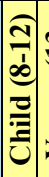 & 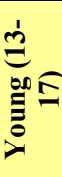 & 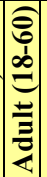 & 产 & ב. & Z & 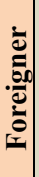 & 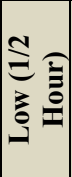 & 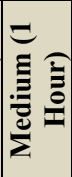 & 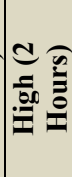 & 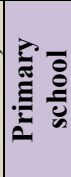 & 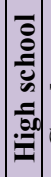 & 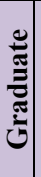 & 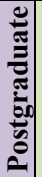 & טل & 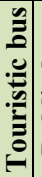 & 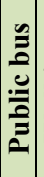 & 先 & 峁 & 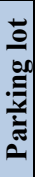 \\
\hline & & & ¿̇ं & לֶ & & & & & & 0 & & & 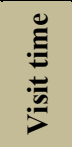 & & & 可 & & & & & 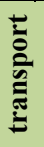 & & 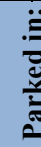 & \\
\hline
\end{tabular}




\begin{tabular}{|c|c|c|c|c|c|c|c|c|c|c|c|c|c|c|c|c|c|c|c|c|c|c|c|c|}
\hline 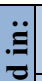 & $\begin{array}{c}\text { म01 } \\
\text { ôu!̣.Iв d } \\
\end{array}$ & ปี & $\nabla$ & $n$ & $N$ & - & 0 & 0 & $\nabla$ & $\sim$ & $m$ & 0 & $N$ & -1 & $m$ & $0 \mid v$ & 6 & 0 & $a / c$ & 0 & 0 & 0 & 0 & $a$ \\
\hline 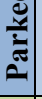 & 1әว.І1S & ב & - & 0 & 0 & 0 & - & 10 & - & 0 & 0 & 0 & 0 & -1 & 0. & -6 & 0 & 0.1 & $-c$ & 0 & 0 & 0 & -1 & 0 \\
\hline 흘 & 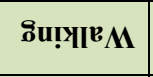 & 吉 & 0 & 0 & 0 & 0 & 0 & 0 & 0 & 0 & 0 & 0 & 0 & 0 & 0 & $0 / 6$ & 0 & 0 & $0 \mid c$ & 0 & 0 & $\theta$ & 0 & 0 \\
\hline (ี & $\operatorname{snq} x_{!}\left[n_{d}\right.$ & ond & $\nabla$ & $m$ & $N$ & in & 0 & 0 & $r$ & 0 & 0 & 0 & - & $6 \mid$ & $m / c$ & $m-$ & - & 0 & 0.0 & 0 & N & 0 & 0 & 0 \\
\hline है & $\begin{array}{c}\text { snq } \\
\text { o!̣s!̣no } L \\
\end{array}$ & bo & 0 & 0 & 0 & 0 & 0 & 0 & 0 & 0 & 0 & 0 & 0 & 0 & 0 & $0 / 6$ & 0 & 06 & $0 \mid \varsigma$ & $\theta$ & 0 & 0 & 0 & 0 \\
\hline$\sum^{0}$ & 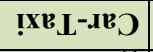 & $\overrightarrow{00}$ & $r$ & 0 & $N$ & - & 으 & 10 & in & $n$ & $m$ & 0 & $N$ & $=$ & $m$. & -10 & $a$ & $0:$ & 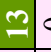 & 0 & 0 & 0 & -1 & $a$ \\
\hline ฮ) & $\begin{array}{c}\text { p18npe.so } \\
-750 \mathrm{~d}\end{array}$ & 志 & 0 & 0 & 0 & 0 & 0 & 10 & 0 & 0 & 0 & 0 & 0 & 0 & 0 & 0.0 & 0 & $\theta$ & 0 & 0 & 0 & 0 & 0 & 0 \\
\hline สำ & әұепре.ग & 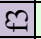 & in & $n$ & 0 & - & $a$ & 0 & $N$ & in & $m$ & 0 & 0 & 음 & 015 & \begin{tabular}{l|l}
0 & 6 \\
\end{tabular} & 르 & 016 & a & 0 & - & 0 & 0 & 6 \\
\hline . & $\begin{array}{c}\text { [00पगS } \\
\text { प } 8 ̊ ! H\end{array}$ & 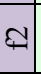 & $\nabla$ & 0 & 0 & $m$ & - & 10 & $\nabla$ & 0 & 0 & 0 & - & $m$ & 0 & $\nabla$ & 0 & 0. & -10 & 0 & $m$ & 0 & -1 & 0 \\
\hline 导 & 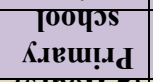 & 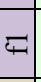 & $N$ & $\nabla$ & $\nabla$ & $\mathrm{N}$ & 0 & 10 & 0 & 0 & 0 & 0 & $N$ & $|\nabla|$ & 6 & 0 & 0 & 0. & $m / c$ & 0 & $m$ & 0 & 0 & $m$ \\
\hline 巳 & $\begin{array}{c}\text { (S.moH Z) } \\
\text { पo̊!H }\end{array}$ & $\hat{\jmath}$ & $a$ & $\infty$ & $m$ & $\nabla$ & 으 & 10 & a & in & $m$ & 0 & 0 & 1 & $\nabla c$ & $m:$ & 의 & $0:$ & 二 & 0 & 6 & 0 & -1 & $r$ \\
\hline 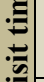 & $\begin{array}{l}\text { (.InoH I) } \\
\text { un!рәК }\end{array}$ & ข & $N$ & -1 & - & 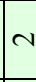 & 0 & 10 & $m$ & 0 & 0 & 0 & $\infty$ & 0 & $N$ & $-1 c$ & 0 & 0 & $\mathrm{~N}$ & 0 & -6 & 0 & 0 & $N$ \\
\hline$>$ & $\begin{array}{c}(\operatorname{InoH} \text { Z/I }) \\
\text { мо T }^{-}\end{array}$ & 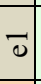 & 0 & 0 & 0 & 0 & 0 & 0 & 0 & 0 & 0 & $\theta$ & 0 & 0 & 0 & 0 & 0 & 0 & 0.0 & 0 & 0 & 0 & 0 & 0 \\
\hline.$\Xi$ & มวนภิ!ฺ.ม & z & N & -1 & 0 & 0 & $m$ & 0 & 0 & 0 & $m$ & 0 & 0 & $m$ & 0 & 0. & $m$ & 0. & $m$ & 0 & 0 & 0 & 0 & $m$ \\
\hline 政 & นвว!хәИ & ชิ & $m$ & $N$ & 0 & 0 & $n$ & 0 & 0 & in & 0 & 0 & 0 & in & 0 & 0.4 & $n$ & 0. & $n$ & 0 & 0 & 0 & 0 & $N$ \\
\hline 0 & [вวоT & $\nabla$ & 6 & 0 & $\nabla$ & 6 & $N$ & 10 & 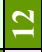 & 0 & 0 & 0 & $m$ & $a$ & 6 & $\nabla 0$ & $N$ & 0. & $n$ & 0 & $\sim$ & 0 & -1 & $\nabla$ \\
\hline & $\begin{array}{c}\text { (ә.лош } \\
\text { ло І9) } \\
\text { К.ләрІ }\end{array}$ & $\tau$ & 0 & 0 & 0 & 0 & 0 & 0 & 0 & 0 & 0 & 0 & 0 & 0 & 0 & 0 & 0 & 0 & 0 & 0 & 0 & 0 & 0 & 0 \\
\hline 品 & $\begin{array}{l}(09-81) \\
\text { भInpV }\end{array}$ & $\overrightarrow{0}$ & 6 & $\nabla$ & 0 & 0 & 음 & 0 & $N$ & in & $m$ & 0 & 0 & 으 & 0. & -10 & a & 0. & 이당 & 0 & 0 & 0 & -1 & 6 \\
\hline & $\begin{array}{l}\text { (LI-EI) } \\
\text { ôno } X\end{array}$ & ปิ & $m$ & $m$ & 0 & 6 & 0 & 0 & 0 & 0 & 0 & 0 & $N$ & $\nabla \mid$ & $\mathrm{N}$ & $m-$ & - & 0. & -10 & 0 & $n$ & 0 & 0 & - \\
\hline & $\begin{array}{l}\text { (2I-8) } \\
\text { pI!पว }\end{array}$ & D & $N$ & $N$ & $\nabla$ & 0 & 0 & 0 & $\nabla$ & 0 & 0 & 0 & 一 & $m \mid$ & $\nabla$ & 0 & 0 & 0 & $\mathrm{~N}$ & 0 & $\mathrm{~N}$ & 0 & 0 & $N$ \\
\hline - & әрвшән & สี| & 0 & $a$ & $\mathrm{~N}$ & $m$ & t & 10 & 6 & $N$ & -1 & 0 & 一 & $\infty$ & $\nabla$ & 0.4 & $n$ & $0 \mid y$ & 6 & 0 & $m$ & 0 & 0 & $n$ \\
\hline లై & गएर्W & స & $=$ & 0 & $N$ & $m$ & 6 & 0 & 6 & $m$ & $N \mid$ & 0 & $N$ & $a \mid$ & $N$ & $\nabla 4$ & $n$ & 01 & NC & 0. & $\nabla$ & 0 & -1 & $\nabla$ \\
\hline & & & च & สี & 0 & O & $\overline{0}$ & 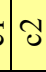 & $\bar{\nabla}$ & ขี & च & $\overrightarrow{0}$ & $\widetilde{\nu}$ & 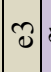 & $=6$ & 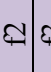 & 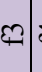 & \pm & $\overline{00} \mid \mathrm{c}$ & कo & की & $\mid \begin{array}{l}\mid t \\
0\end{array}$ & ב & ป \\
\hline & $\underset{\|}{\stackrel{N}{N}}$ & & $\frac{0}{\Sigma}$ & 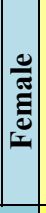 & 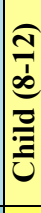 & 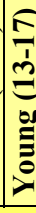 & 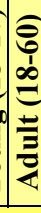 & $\frac{2}{2}$ & 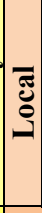 & 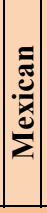 & 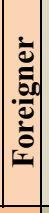 & 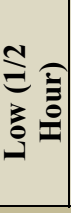 & 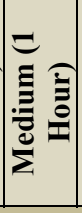 & 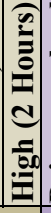 & 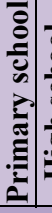 & 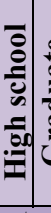 & 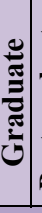 & 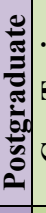 & שֶ. & 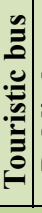 & 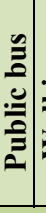 & 照 & 离 & 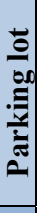 \\
\hline & 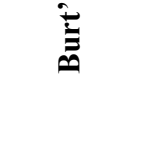 & & 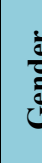 & 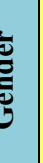 & & & $\overbrace{\infty}^{\infty}$ & & & ह0 & & & 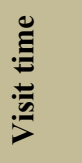 & & & 氕 & d & & & 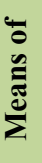 & 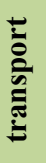 & & . & Ė \\
\hline
\end{tabular}




\begin{tabular}{|c|c|c|c|c|c|c|c|c|c|c|c|c|c|c|c|c|c|c|c|c|}
\hline $\begin{array}{l}. \Xi \\
\bar{\sigma}\end{array}$ & $\begin{array}{c}\text { 10I } \\
\text { su!Y.IEd }\end{array}$ & 구 & $N-$ & 40 & 0 & n & -1 & $N-$ & $-c$ & 0 & - & $N$ & $-16 \quad-2$ & 0.0 & $N e$ & $m$ & 0 & & 0 & $p \mid \infty$ \\
\hline \begin{tabular}{|l|}
$\tilde{\Xi}$ \\
$\triangleq$
\end{tabular} & 2.IIS & $\exists \mathrm{c}$ & $N-$ & 10 & 0 & $m$ & 0 & m & 00 & $0-$ & $-1 N$ & 0 & 0 & & $N C$ & $m$ & 0 & 0 & 0 . & $n$ \\
\hline 힘 & II & ton. & $-\gamma$ & 0 & 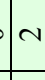 & - & 0 & $m$ & 0 & 0 & N & - & 0 . & $m$ & 0 & 0 & 0 & 0 & $m$ & \\
\hline 量 & snq $\operatorname{p!Iq}_{\mathbf{d}}$ & 30. & $-\infty$ & 0 & - & r & - & $\nabla$ & $n$ & 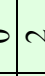 & - & 6 & $a$ & o. & -0 & 0 & 0 & $a$ & 0 & o \\
\hline $\begin{array}{l}-4 \\
\vdots \\
\vdots \\
\end{array}$ & $\begin{array}{c}\text { Snq } \\
\text { ग!̣s!uno } \mathbf{L} \\
\end{array}$ & bo & 00 & 0 & 0 & 0 & 0 & 0 & 0 & 00 & o & 0 & $0 / 6$ & 00 & 00 & 0 & 0 & 0 & 0 & \\
\hline$\sum$ & 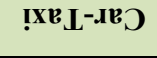 & bol 4 & $n \pi$ & 0 & 0 & $r$ & - & $r$ & $-c$ & $p$ & $m$ & 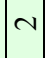 & -0 & $m$ & $+c$ & $\infty$ & 0 & 0 & ○ & $?$ \\
\hline$\left|\begin{array}{|c}0 \\
0 \\
0\end{array}\right|$ & $-150 d$ & 氙 & 00 & 0 & 0 & 0 & 0 & 00 & 00 & 0 & 0 & 0 & $0 \mid c$ & 00 & $0 \mid e$ & 0 & 0 & 0 & 00 & olo \\
\hline$\overline{\underline{\pi}}$ & әұепре.19 & $\notin 0$ & $n e$ & 0 & 0 & in & 0 & + & $-c$ & 0 & m & $N$ & 0.0 & 0.1 & in 0 & $\theta$ & 0 & - & $\circ$ & v \\
\hline . & & $\$$ & $+\infty$ & 0 & $m$ & $a$ & 0 & $\infty$ & $+c$ & pr & $\mathrm{N}$ & $n$ & $0 \mid:$ & 기이 & 0 & $m$ & 0 & 0 & $m$ & 10 \\
\hline 홍 & $\kappa$ & $\overline{\mid} \mid-$ & $-c$ & $\mathrm{~N} O$ & 0 & - & $N$ & $\mathrm{~N}$ & $-c$ & c & T & $n$ & $m$ & 0 & $0 / 0$ & - & 0 & & 00 & \\
\hline 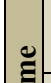 & $\begin{array}{r}\text { (S.Ino } \\
\mathbf{4} 8 \\
\end{array}$ & ช. & & 0 & - & r & - & $r$ & $N e$ & 0 & 0 & a & $\left.N\right|^{4}$ & $n$ & $\mathrm{~N} / \mathrm{C}$ & 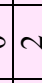 & 0 & 0. & $-c$ & 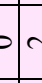 \\
\hline 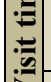 & I) & ขै & $m /$ & 0 & $p$ & $m$ & - & + & $N C$ & 0 & b & 0 & -10 & 40 & $m / c$ & $m$ & 0 & . & $N / c^{2}$ & v. \\
\hline$>$ & (.In & $\overline{0}$ & $N m$ & 0 & 0 & in & 0 & $m$ & $N C$ & on & 0 & 0 & 0.4 & $n$ & $0 / 0$ & $m$ & 0 & $\sim$ & $0 .-13$ & - o \\
\hline$\Xi$ & $\mathbf{I}$ & 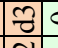 & 00 & 0 & 0 & 0 & 0 & 0 & 0.5 & 0 & $\rho$ & 0 & $0 / 5$ & 0.5 & 0.0 & 0 & 0 & 0 & 0.5 & 0 \\
\hline 番 & Ir & శैc & $4 \curvearrowright$ & o 0 & 0 & in & - & 0 & $6 / 5$ & or & v & 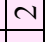 & -1 & +7 & -10 & $0-$ & 0 & in & $0 / 5$ & - - \\
\hline & $\mathbf{0}^{\prime}$ & $\bar{\nabla}$ & $n \sigma$ & 10 & $m$ & - & - & \pm & 016 & 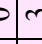 & c & $r$ & No & $\infty$ & $\nabla 0$ & $r$ & 0 & $\nabla$ & $m / c$ & 2 \\
\hline & & $\mathcal{O}$ & - & 0 & 0 & 0 & $N$ & - & - & 0 & & -1 & 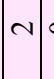 & 00 & 00 & $0-$ & 0 & & 00 & 5 \\
\hline 总 & & $\overline{0}$ & $n \subseteq$ & 0 & 0 & $\cong$ & 0 & 으. & $n$ & D ir & s & $r$ & -1 & & $n$ & 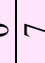 & 0 & - & $-c$ & $n$ \\
\hline & & $\mathcal{D}-$ & $\rightarrow \sim$ & $\mathrm{N} O$ & $m$ & 0 & 0 & $m$ & 0.0 & 0 & $N$ & - & 0 & $m c$ & 00 & 00 & 0 & - & $N$ & D.c \\
\hline & & 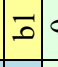 & 00 & $b e$ & 0 & 0 & 0 & 0 & 0.0 & | & & p & 0 & $0 / 5$ & 0.0 & 0 & 0 & & 00 & 4 \\
\hline 힣 & Uo & $\approx$ & $0 \cong$ & 0 & $N$ & 으 & - & a & $\nabla$ & & & $r$ & $\sim$ & $\infty$, & $m c$ & $b m$ & 0 & $\infty$ & $N$ & - \\
\hline 准 & & $\bar{\pi}$ & $r o$ & 0 & -1 & in & - & in & $N$ & 0 & $m$ & $\sim$ & - & $\nabla$ & $N C$ & $b n$ & 0 & & $-c$ & v \\
\hline \multirow{3}{*}{\multicolumn{2}{|c|}{ 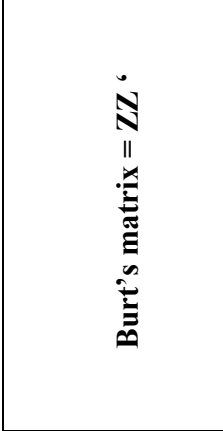 }} & & $\bar{\pi}$ & $y=$ & 5 & $\overline{0}$ & ช & $\bar{\nabla}$ & శึ) & $\theta$ & ช & 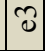 & 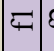 & 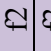 & $\oiiint 4$ & \pm & of & 30 & 茄 & $=$ \\
\hline & & & 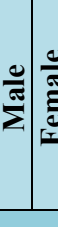 & 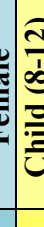 & 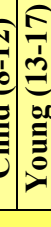 & 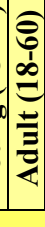 & $\frac{\pi}{\frac{\pi}{v}}$ & 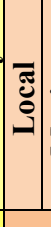 & : & 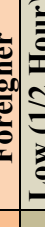 & 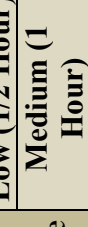 & 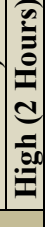 & 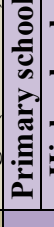 & 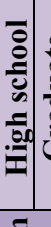 & 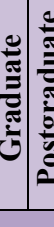 & لَّ & 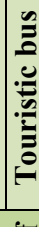 & 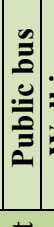 & . & 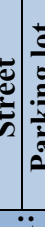 \\
\hline & & & 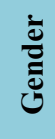 & & & & & & & & 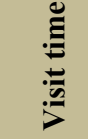 & & & & & & & 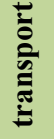 & & 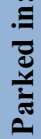 \\
\hline
\end{tabular}




\begin{tabular}{|c|c|c|c|c|c|c|c|c|c|c|c|c|c|c|c|c|c|c|c|c|c|c|c|c|}
\hline ت. & $\begin{array}{c}101 \\
\text { su!y.лвd }\end{array}$ & ฮี & $N$ & $N$ & 0 & 0 & $m$ & -1 & $\nabla$ & 0 & 0 & $m$ & - & 0 & 0 & $N$. & -1 & - & $\nabla$ & 0 & 0 & 0 & 0 & $\nabla$ \\
\hline$\approx$ & คәว.ISS & ב & 0 & -1 & 0 & 0 & -1 & 0 . & - & 0 & 0 & - & 一 & 0 & 0 & -10 & 0 & 0 & -1 & 0 & 0 & 0.1 .12 & -1 & 0 \\
\hline 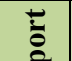 & 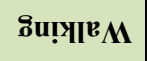 & $\mid$ & $N$ & 0 & 0 & -1 & 0 & $-c$ & $N$ & 0 & 0 & $v$ & 0 & 0 & - & $-c$ & 0 & 0 & 0 & o) & 0 & $N$ & 0 & 0 \\
\hline స్ & snq $\operatorname{s!l} n_{d}$ & की & $\nabla$ & - & 0 & -1 & - & $m$ & $a$ & $N$ & 0 & $m$ & $n$ & $m$ & $\nabla$ & 0. & - & 0 & 0 & o & $=$ & 0 & 0 & 0 \\
\hline : & $\begin{array}{c}\sin 4 \\
\text { ग!ฺs!.nno } L\end{array}$ & ob & 0 & 0 & 0 & 0 & 0 & 0 c & 0 & 0 & 0 & 0 & 0 & 0 & 0 & 0 & 0 & 0 & 0 & $\theta$ & 0 & 0 & 0 & 0 \\
\hline$\sum^{e}$ & 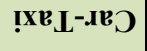 & 50 & $N$ & $n$ & 0 & 0 & $n$ & 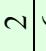 & 6 & - & 0 & $\nabla$ & $\nabla$ & 0 & - & $\nabla$ & -1 & - & N & 0 & 0 & 0 & - & $\nabla$ \\
\hline$\overline{0}$ & 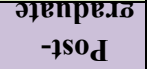 & 进 & - & 0 & 0 & 0 & -1 & 0 & - & 0 & 0 & - & 0 & 0 & 0 & 0 & 0 & - & - & 0 & 0 & 0 & 0 & - \\
\hline $\bar{\Xi}$ & әџепре.গ & $\notin$ & $N$ & 0 & 0 & 0 & N & 0 & 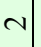 & 0 & 0 & 0 & $\sim$ & 0 & 0 & o.e & N & 0 & - & 0. & - & 0 & 0 & - \\
\hline • & $\begin{array}{c}\text { [00पगS } \\
\text { Чภิ!H }\end{array}$ & $\nsubseteq$ & $N$ & $a$ & 0 & $N$ & $\infty$ & -1 & 으 & -1 & 0 & - & $\nabla$ & -1 & 0 & $=$ & 0 & 0 & $\nabla$ & 인 & 0 & 一 & - & $v$ \\
\hline ت્1 & 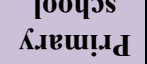 & E & $m$ & $m$ & 0 & 0 & -1 & $n$ & $\nabla$ & N| & 0 & -1 & $m$ & $N$ & 6 & 0 & 0 & 0 & 一 & 0 & $\nabla$ & 一 & 0 & 0 \\
\hline$=$ & $\begin{array}{c}(\text { S.InoH Z) } \\
\text { पo̊!! }\end{array}$ & \% & 0 & $m$ & 0 & 0 & $N$ & -1 & $m$ & 0 & 0 & 0 & 0 & $m$ & $\mathrm{~N}$ & $-c$ & 0 & 0 & 0 & 0 & $m$ & 0 & 0 & 0 \\
\hline$=$ & un!рәК & ชิ & in & $\nabla$ & 0 & 0 & 0 & $m$ & 6 & $m$ & 0 & - & $a$ & 0 & $m$ & $\nabla c$ & N & 0 & $\nabla$ & 인 & $n$ & 0 & - & - \\
\hline$>$ & $\begin{array}{c}\text { (moHz } z / I) \\
\text { MOT }\end{array}$ & $\overline{0}$ & $m$ & 6 & 0 & $N$ & $n$ & $N$ & $a$ & 0 & 0 & a & 一 & 0 & -1 & - & 0 & - & $\nabla$ & 인 & $m$ & N & - & $m$ \\
\hline & 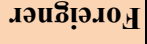 & $\eta$ & 0 & 0 & 0 & 0 & 0 & 0 & 0 & 0 & $\theta$ & 0 & 0 & 0 & 0 & 010 & 0 & 0 & 0 & 0 & 0 & 0 & 0 & 0 \\
\hline 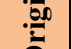 & ивว!хәИ & ขี & - & $N$ & 0 & 0 & - & $\sim$ & 0 & $m$ & 0 & 0 & $m$ & 0 & N & $-c$ & 0 & 0 & 一 & o & $N$ & 0 & 0 & 0 \\
\hline & [вวо Т & $\bar{\nabla}$ & 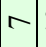 & 으 & 0 & $N$ & 二 & $\nabla$ & 드. & 0 & 0 & $a$ & 6 & $m$ & $\nabla$ & 잉 & N & - & 6 & 0 & $a$ & N. & -1 & $\nabla$ \\
\hline & 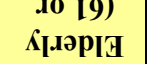 & ชิ & $m$ & $m$ & 0 & 0 & o & 6 & $\nabla$ & N & 0 & $v$ & $m$ & - & $n$ & - & 0 & 0 & $N$ & 0 & $m$ & 一 & 0 & - \\
\hline 8 & $\begin{array}{l}\text { (09-8I) } \\
\text { भInpV }\end{array}$ & $\overline{0}$ & $\nabla$ & $\infty$ & 0 & 0 & ㄱ. & 0 & $=$ & -1 & 0 & $n$ & 6 & N. & -1 & $\infty \mid c$ & 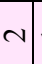 & - & in & 인 & r & 0 & -1 & $m$ \\
\hline & $\begin{array}{l}(L I-\varepsilon I) \\
\text { ôuno } \lambda\end{array}$ & 지 & - & - & 0 & $\mathrm{~N}$ & 0 & 0 & v & 0 & 0 & v & 0 & 0 & 0 & $\mathrm{~N}$ & 0 & 0 & 0 & 0 & -1 & 一 & 0 & 0 \\
\hline & $\begin{array}{l}(21-8) \\
\text { р!!Чว }\end{array}$ & - & 0 & o & $\theta$ & 0 & 0 & 0 & 0 & 0 & 0 & 0 & 0 & 0 & 0 & 0 & 0 & 0 & 0 & 0 & 0 & 0 & 0 & 0 \\
\hline ᄒ & ившом & สิ & 0 & $\stackrel{\sim}{=}$ & 0 & - & $\infty$ & $m$. & 으 & $N$ & 0 & 6 & $\nabla$ & $m$ & $m$ & $a$ & 0 & 0 & in & 0 & N & 0 & - & $N$ \\
\hline 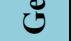 & ивW & $\pi$ & $\infty$ & 0 & 0 & - & $\nabla$ & $m$ & - & - & 0 & $m$ & $n$ & 0 & $m$ & $\mathrm{~N}$ & 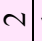 & - & $N$ & 0 & $\nabla$ & $\mathrm{N}$ & 0 & $N$ \\
\hline & \multirow{3}{*}{ 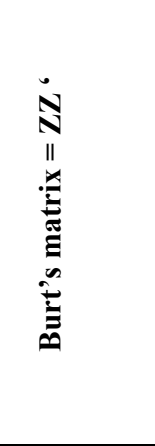 } & & $\bar{\pi}$ & สี & 이 & ปิ & $\overline{0}$ & ช & 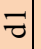 & 기 & m & 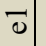 & ๙ & 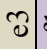 & 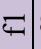 & ๘) & 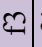 & ष্t & $\overrightarrow{00}$ & ob & రి. & ${ }_{0}$ & $\Xi$ & ปี \\
\hline & & & $\frac{\frac{0}{\sigma}}{\Sigma}$ & 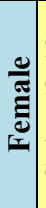 & 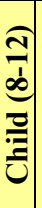 & 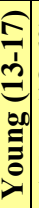 & 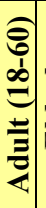 & 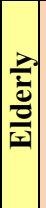 & 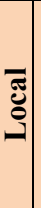 & 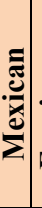 & 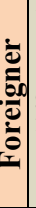 & 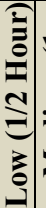 & 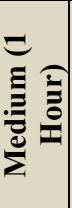 & 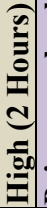 & 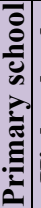 & 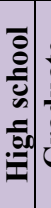 & 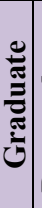 & 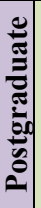 & | & 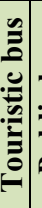 & 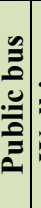 & | & 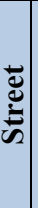 & 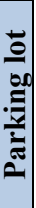 \\
\hline & & & & כ. & & & |20 & & & 范 & & & 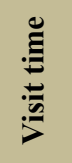 & & & 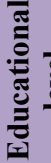 & ঠ & & & & 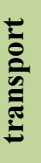 & & $\Xi$ & $\begin{array}{l}y \\
y \\
z \\
z\end{array}$ \\
\hline
\end{tabular}


Most of the people who visited the Museum were adults from 18 to 60 years old, but also there were at the place young people (13-17 years old) and kids (8-12 years old) from primary schools. Besides, there's a fact that elderly people don't visit too often the Museum, and it doesn't depend on the general topics exposed in the works of art, but in the lack of accessibility for them.

Most of the visitors to the Museum spent more than an hour in the building. Even though it's a national museum - actually it's a regular size museum - a complete visit usually takes from two to four hours.

Also, it's interesting to notice graduate people spent two hours or more in the Museum. No postgraduate visitors were found during the research. Parking lots played an important role for the visitors to the Museum. Since public buses weren't that efficient in the city, visitors preferred to travel by car and park close to the Museum. This situation has become a problem in the historic centre; because some historic houses are now being torn down to replace architecture with brand new parking lots so visitors to the commercial shops and iconic historic places can park.

Public buses run frequently from different sides of the city to historic sites in Aguascalientes. $35 \%$ of the inquired people took a public bus to get to the National Museum of Death, and $65 \%$ preferred to use a private car or a taxi (see Table 2). The culture of public transit still has to be improved in the city. Also, public transport sometimes is insecure because they use old buses and some of them look worn out. Public buses are private in Aguascalientes and sometimes government subsidises them with low budgets.

The main square of the city was originally the first square designed by the Spaniards in the state in the $16^{\text {th }}$ century. The place is a landmark for the citizens and has become a visitors' favourite place in the city. The main square has different types of visitors depending on the weekday. From Monday to Saturday it's common to find elderly people talking under the shades of the trees. Also, there are visitors from some other states of Mexico, and some citizens of Aguascalientes go to the local government offices near the square. During the weekends it turns into a place where families go and enjoy days of fun due to the activities. The government occasionally organizes concerts or different types of amenities for the citizens.

According to the questionnaires applied in the main square, most of the visitors were adult people (from 18 to 60 years old) or the elderly (61 or more years old); this is $85 \%$ of the visitors on a weekday. Also $70 \%$ of the visitors were local, and the others came from different states of the country. $45 \%$ of the visitors spent two hours or more at the place. The results demonstrated that $60 \%$ of the visitors had a high school level and again, just like the other historic places analysed in this paper, no postgraduate visitors were found.

In this case $45 \%$ of the visitors came to the main square by public bus; $40 \%$ arrived by car or by taxi; and only $15 \%$ came walking.

The people who came walking lived close to the historic centre, even though a few historic houses still stand. Also, the people who drove a car to the historic centre parked in the streets or in parking lots near the main square.

The historic France Hotel is located in front of the main square in the historic centre. In fact, it is very easy to get there by public bus. The collected data from questionnaires that were applied at the historic France Hotel (see Table 4) showed that $60 \%$ of the visitors were women, and $40 \%$ were men. $60 \%$ of the visitors were adults from 18 to 60 years old. Also most of the visitors $(85 \%)$ were local; on the other hand, $15 \%$ came from some other Mexican states. One of the reasons of this local preference is the restaurant situated inside the France Hotel and the commercial shop located on the first story of the historic building. The people who preferred to visit the place were basically adults with high school level. Even though 
most of them preferred to stay at the place less than half an hour some of them preferred two hours visit time. The low time visitors went to the place to shop and the high time visitors ate at the restaurant.

$55 \%$ of the visitors preferred to take a bus to arrive to the historic place, and $20 \%$ of the visitors searched for a parking lot to park. Only $5 \%$ of the visitors looked for a spot to park on the streets. If we observe the categories of the variable: Means of transport and how they are associated to the other categories it can be inferred that $35 \%$ of the people are adults who took a public bus to arrive to the historic place. Anyone took a touristic bus, but $85 \%$ of the visitors were local and the half of them preferred to take a bus.

Even though we can observe the loss of architecture by the construction of new parking lots, public transportation can be improved to offer a better service to arrive to the historic centre. The design and installation of an electric train would be an attractive transportation offer to the visitors of the historic centre. Also, this might stop the loss of historic architecture in the city of Aguascalientes.

\section{DISCUSSION}

In the discussion about the data obtained from the interviews at the Cathedral place (see Table 1 ), it can be inferred that means of transport is one of the most important variables. Since urban transport is a significant topic nowadays, the analysis of the way people arrive to the main historic places of the city is valuable. So, the first results of the Burt's table had to do with gender, and the fact of taking a car or a taxi was more related to women. This means seven women over two men preferred to arrive driving a car or taking a taxi to get to the main Cathedral. Any of the interviewed people arrived in a touristic bus, and a few of them arrived walking.

Besides, only four women and four men took a bus to arrive to the Cathedral place; even though parking on the streets is more difficult nowadays because there are more cars circulating in the city. The population in the city of Aguascalientes is approximately 800,000 people in 2018, and the government reported in July of this year 522,000 vehicles in the State of Aguascalientes. $70 \%$ of this amount circulates in the city. This means 365,400 vehicles. So, there's almost a car for every two persons in the city. Comparing Aguascalientes with Mexico City, for example, this amount is too high. The average in Mexico City is one car for every five vehicles.

Hence, the amount of cars circulating in the historic centre of Aguascalientes make more difficult to find a spot to park in the streets when someone drives, so, in general, most of the people who visit and drive to historic places have to look for a parking lot. Some people preferred to drive a car to go to the historic centre instead of taking a bus. Rześny says the expansion of urban structures, the increase in the number of urban population and related flows gradually contribute to the deterioration of life quality in urban areas [2], and this is the case of the city of Aguascalientes, with so many difficulties in local urban transportation.

Nowadays, accessibility devices - like elevators - have become a problem when restoring historic buildings because they're expensive and multiple restoration designs skip their integration. This is the case of the National Museum of Death. Also, the lengths of the pathways to observe some of the exhibitions are too long, with no places to take walk breaks and seat for a while. The accessibility devices are useful and their integration in restoration projects should be included.

The means of transportation used by the visitors to the museum were cars or taxis (the most frequent), and no visitors used a touristic bus. The touristic buses in the city of Aguascalientes usually circulate through historic places, but they never stop and wait for the 
people to get off to enter buildings or museums and then return. They are more like a street sightseeing experience, including public spaces. And they're usually decorated like historic tramways. Most of the respondents in the research were inhabitants from the same city of Aguascalientes. But 25\% came from some other states of Mexico, and three persons were foreigners. This situation is different from the matrix analysis at the main Cathedral, and foreigners showed interest in learning about the Mexican perspective of the death symbols.

The Burt's matrix demonstrated that the main square is not attractive to young people. If the main square is the oldest place in town and young people don't visit it then, the knowledge and attitudes to historic preservation among youth still need special attention in Mexican education. In fact, young people don't want to live in the historic centre because they think it's an old-fashioned place and the conditions of buildings aren't what they really expect. Furthermore, the influence of modern mobility is strong and young couples prefer to buy a car and live in a suburb, rather than living in a historic place where there's no place to park their vehicles.

Also, there's a new social phenomena in the historic centre that has to do with youth. In spite of the lack of interest for young people to live in the historic centre, there are new attractions for them like new bars and nightclubs. Even though the questionnaires for this research were applied to collect data in the morning time and at noon, the increasing nightlife in the city centre is obvious. So, young people don't go too often to public spaces during daytime, nor do they like to live in the historic centre, but they prefer to spend there sometime at night to have fun.

It's time to improve city infrastructure and change the travel behaviours demonstrated in this research. Guerra and Cervero say Transit reduces traffic congestion and tailpipe emissions when it draws potential motorists, particularly single-occupant drivers, to train and buses [3].

A new light rail transit system (LRT) could be designed and installed in the historic centre and connected to the old railroad station (see Fig. 3), even though such station is now a museum and part of the Tres Centurias Park. Cargo railroad trains are now using the tracks. But in a near future they could be used -through a correspondence station- with passenger trains. The new LRT could be named "El San Marqueño" because of the legendary San Marcos National Faire that takes place in Aguascalientes every year. Also, now we can observe the artistic vision in trains, as Banyas mentions: Transit agencies across the nation are applauding the success of public art in their LRT systems. Public enthusiasm is high, and anecdotal evidence points to a reduction in vandalism and an increase in stewardship for neighbourhood stations. Using a tiny fraction of project budgets, artists are adding a sense of beauty, delight, curiosity, and identity to stations and systems alike [4]. Also, in the city of Aguascalientes' planning it's convenient to include a Transit Oriented Development (TOD), but not only focusing in cars or public buses, but in electric trains also. Furlan and AlMohannadi argue that TODs can increase access to public transportation, work places, educational institutions and other facilities by promoting transportation options to households [5].

Urban growth has become a problem in Aguascalientes and certain avenues keep heavy traffic in rush hours. When referring to traffic operation with recurrent congestion Szele argues that the unfairness of traffic operation is based on a contradiction between individual and social interests [6], but individual interests can be changed with a contemporary traffic consciousness among car drivers. 


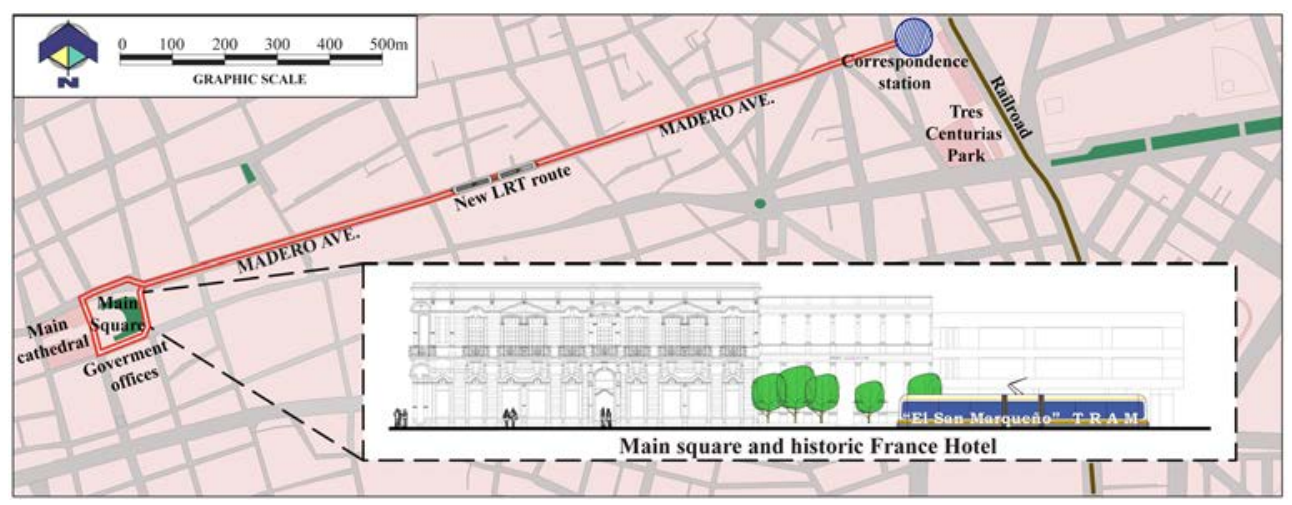

Figure 3: A light rail transit system (LRT), with floor-level boarding, could be a good option to communicate the Tres Centurias Park with the city centre. (Source: Design and drawing by Alejandro Acosta Collazo, Víctor Gerardo González González and Jéssica Alejandra Rodríguez Torres, 2018.)

\section{CONCLUSIONS}

The multidimensional analysis was used efficiently to show similar relationships or preferences hidden in the collected data. After analysing the results of the research, it's recommended to promote tourism in the historic centre abroad. National visitors, foreigners, local visitors and the government could contribute to create new generation mobility systems for historic places like an LRT.

It's convenient to increase architecture and restoration students' skills in schools to design for accessibility. This would enable people with disabilities to visit and enjoy museums of interest, for example.

The public transportation system requires new pathways and efficient transfer routes to improve urban mobility. Chowdhury and Ceder mention that transfers play a key role in an integrated PT [public transport] system. Creation of transfer routes in an integrated PT network has also been seen to make the service more efficient and reliable [7].

The government started protecting some historic buildings in 1990, so the owners had to decide whether they stayed living in such houses or renting them. Besides, most of the nightclubs installed in the historic centre rented the building they use. Nightclubs moreover had a negative effect in the image of the streetscapes, especially at night. Then, loss of habitat became a preservation problem in the city centre. City planning can contribute to historic preservation, an owner of a historic building can also, but sometimes in Mexico people expect the resources designated to historic preservation should come from the government. This is a wrong appreciation. Even though the owners of historic buildings aren't allowed to tear them down they could help to preserve them if they understood their importance as cultural icons in the city.

At present time there are less historic buildings compared with early years of this Millennium. Somehow the loss of architecture was influenced by a lack of regulations in urban mobility. Also, the historic buildings protected by regulations against structural damages, for they are better preserved.

Every year there's a huge event in the city of Aguascalientes, named San Marcos National Fair. It takes place during the months of April and May. The almost one million people city receives seven million visitors (the largest fair of Mexico) in a length of three weeks. The 
amenities include a beauty contest (Queen of the Fair), bullfighting, cockfighting, charreadas (rodeos), livestock shows, attractions, a casino, concerts, cultural activities, etc. As a result, urban mobility becomes complicated at fun fair times. And the problem isn't only caused to mobility; but also to hotels capacity; to public spaces; to San Marcos Quarter, and to the surrounding inhabitants who have to deal with drinking places and happy people (drunk) around the historic centre at night-time.

The city of Aguascalientes - with sunny days in April and rainy days in May - receives the Fair every year. The Fair changes the sceneries in the main square, the historic France Hotel, the National Museum of Death, the main Cathedral and the whole historic centre. The Fair also changes the transportation systems.

A concern detected on the writing of this paper is how the government could make the historic centre a sustainable place to visit; without allowing tearing down historic buildings; without affecting tourism and including an efficient urban mobility. Schoeman says sustainability within spatial systems refers to the optimization of planning, resources and activities so as to protect the environment through the scientific design, approaches and accountability. This includes transportation, urban form, quality in the utilization of the environment, food production, land and water resources [8]. So, finding solutions for urban mobility is a complex problem. The preservation of the historic centre requires specific studies about urban mobility, and the whole city has to do with it. Sitányiová and Masarovičová mention the larger the city, the greater its complexity and the potential for disruption. Often this complexity is not effectively managed, especially the preparation of necessary new policies, such as the promotion of more energy-efficient vehicles or alternative fuels, support for public transport, transport demand management or anti-sprawl legislation [9].

The historic centre of Aguascalientes is not like a European historic centre. There are a lot of changes in the streetscapes every two or three years here. Commercial shops are becoming popular in the historic places, and Mexican folk and Mexican habits are in quotidian life and events. If someone gets on a bus, they can notice them; this is, on the way drivers circulate through the city and on the way drivers decorate their own bus cubs. Rushing hours are complicated in the historic centre, basically because of schools and drivers who cross the historic centre to go to the other side of the city.

The multiple correspondence analysis method used in this paper showed the important connection between mobility and the historic centre. In order to attenuate the loss of architecture, it's convenient to set new regulations on the way a building can be protected and preserved. But schools play an important role on cultural preservation. So, if we teach our kids on time to identify and enjoy the historic centres they can begin to observe the valuable built surroundings.

Timetables should be followed and could be posted in bus stops to make a better public bus system in the city. As well as including newer public buses, with less polluting engine systems.

Antoniou and Tyrinopoulos mention many urban touristic areas around the world face numerous problems caused by the seasonality of transport demand, meaning the predictable or unpredictable variations in demand for transport services imposed by other sectors; in this case tourism. Such problems are congestion in the major transport routes, damage of the transport infrastructure, severe environmental pollution, energy loss, increased travel times, damage to the natural environment, low quality services provided to the visitors and harms to the historical sites [10]. This is the case of San Marcos Fair. Touristic routes should be included in city planning in order to consider the impact of tourism in the historic centre. Also, city planning should include the principles of sustainability. Schoeman argues the UN 
identified the urban mobility challenge and more specifically the sustainability challenge to pivot on the integration of land use and transportation planning; social dimensions and reality; environmental dimensions; economic dimensions and institutional and governmental roles and responsibilities [11]

The research strategy in this paper demonstrated that qualitative tools are as efficient as quantitative tools. Even though, exact sciences prefer the use of absolute precision strategies. In the research of historic places, qualitative techniques (or combined techniques) become useful. About the process of building theory from case study research, Huberman and Miles argue that an investigator may move from cross-case comparison, back to redefinition of the research question [12] so in qualitative research the data obtained from the inquired people should be compared with other opinions and should be analyzed with the scope of appropriate methodology.

Sometimes people tear down historic buildings; therefore, the government must have strict preservation controls. A good solution to preserve historic buildings is that government can take care of restoring the main façades in the streetscapes; just us the owners of the buildings can take care of restoring the interiors of their houses.

Conclusively, the results of the research demonstrate that it looks like owning historic buildings is not a good business - inferred by the loss of historic architecture. Also, the historic houses in the historic centre are expensive, yet some investors have from six to ten buildings.

New transport routes - including less polluting systems - are convenient to the city of Aguascalientes. Chowdhury, Rajaobelison and Ceder say that globally, it has been acknowledged that to relieve congestion in metropolitan cities and to reduce greenhouse gas emissions, modal switch from single occupancy private car use to public transport is vital [13], so the inclusion of a light rail transit system is good option for the city of Aguascalientes and its historic centre.

\section{ACKNOWLEDGEMENTS}

I thank my research assistant Jéssica Alejandra Rodríguez Torres. I also thank the students: Fernando Jesús May Vázquez, Ricardo Daniel Alfaro Ake, Víctor Gerardo González González and Alejandro Acosta Colunga. Appreciation is extended to my friend and researcher: Marco Alejandro Sifuentes Solís, and finally to my professor Jorge Raúl Pérez Gallardo.

\section{REFERENCES}

[1] Camiz, S. \& Gomes, G.C., Alternative methods to multiple correspondence analysis in reconstructing the relevant information in a Burt's table. Pesquisa Operacional, 36(1), pp. 23-44, 2016. DOI: 10.1590/0101-7438.2016.036.01.0023.

[2] Rześny Cieplińska, J., Transport organizers' integrating role in city logistics. International Journal of Transport Development and Integration, 2(1), pp. 30-38, 2018. DOI: 10.2495/tdi-v2-n1-30-38.

[3] Guerra, E. \& Cervero, R., Cost of a ride: The effects of densities on fixed-guideway transit ridership and costs. Journal of the American Planning Association, 77(3), pp. 267-290, 2011. DOI: 10.1080/01944363.2011.589767.

[4] Banyas, R., The transit landscape. Public Art Review, 13(2), p. 16, 2002.

[5] Furlan, R. \& AlMohannadi, M., Light rail transit and land use in Qatar: An integrated planning strategy for Al-Qassar's TOD. International Journal of Architectural Research: ArchNet-IJAR, 10(3), p. 170, 2016. DOI: 10.26687/archnet-ijar.v10i3.1020. 
[6] Szele, A. \& Kisgyörgy, L., Traffic operation on a road network with recurrent congestion. WIT Transactions on the Built Environment, vol. 179, WIT Press: Southampton and Boston, pp. 233-244, 2018.

[7] Chowdhury, S. \& Ceder, A., Definition of planned and unplanned transfer of public transport service and user decisions to use routes with transfers. Journal of Public Transportation, 16(2), pp. 1-20, 2013. DOI: 10.5038/2375-0901.16.2.1.

[8] Schoeman, C.B., International perspectives on transportation and Urban form integration. International Journal of Transport Development and Integration, 1(1), pp. 1-15, 2017. DOI: 10.2495/tdi-v1-n1-1-15.

[9] Sitányiová, D. \& Masarovičová, S., Development status of sustainable urban mobility plans in European Union new member states. International Journal of Transport Development and Integration, 1(1), pp. 16-27, 2017. DOI: 10.2495/tdi-v1-n1-16-27.

[10] Antoniou, C. \& Tyrinopoulos, Y., Factors affecting public transport use in touristic areas. International Journal of Transportation, 1(1), pp. 91-112, 2013. DOI: 10.14257/ijt.2013.1.1.06.

[11] Schoeman, C.B., Spatial planning, transportation planning and environmental management policy informing strategic planning in support of modelling as integrative instrument in developing countries. International Journal of Transport Development and Integration, 1(2), pp. 182-193, 2017. DOI: 10.2495/tdi-v1-n2-182-193.

[12] Huberman, A.M. \& Miles, M.B., The Qualitative Researcher's Companion, Sage Publications: London, p. 28, 2002.

[13] Chowdhury, S., Rajaobelison, D. \& Ceder, A., Car users' willingness to use public transport routes involving transfers. International Journal of Transportation, 4(3), pp. 63-72, 2016. DOI: 10.14257/ijt.2016.4.3.05. 